\title{
Extracting Information about the Electronic Quality of Organic Solar-Cell Absorbers from Fill Factor and Thickness
}

\author{
Pascal Kaienburg, ${ }^{1}$ Uwe Rau, ${ }^{1}$ and Thomas Kirchartz ${ }^{1,2, *}$ \\ ${ }^{1}$ IEK5-Photovoltaik, Forschungszentrum Jülich, 52425 Jülich, Germany \\ ${ }^{2}$ Faculty of Engineering and CENIDE, University of Duisburg-Essen, \\ Carl-Benz-Straße 199, 47057 Duisburg, Germany
}

(Received 18 February 2016; revised manuscript received 4 July 2016; published 4 August 2016)

\begin{abstract}
Understanding the fill factor in organic solar cells remains challenging due to its complex dependence on a multitude of parameters. By means of drift-diffusion simulations, we thoroughly analyze the fill factor of such low-mobility systems and demonstrate its dependence on a collection coefficient defined in this work. We systematically discuss the effect of different recombination mechanisms, space-charge regions, and contact properties. Based on these findings, we are able to interpret the thickness dependence of the fill factor for different experimental studies from the literature. The presented model provides a facile method to extract the photoactive layer's electronic quality which is of particular importance for the fill factor. We illustrate that over the past 15 years, the electronic quality has not been continuously improved, although organic solar-cell efficiencies increased steadily over the same period of time. Only recent reports show the synthesis of polymers for semiconducting films of high electronic quality that are able to produce new efficiency records.
\end{abstract}

DOI: 10.1103/PhysRevApplied.6.024001

\section{INTRODUCTION}

One of the main drivers of the increase in organic solar-cell efficiencies from early papers like the 2001 report by Shaheen et al. [1] on a $2.5 \%$ poly [2-methyl,5-(3*, 7** dimethyloctyloxy)]-(p-phenylene vinylene):[6,6]-phenyl $\mathrm{C}_{61}$-butyric acid methyl ester (MDMO-PPV: $\mathrm{PC}_{61} \mathrm{BM}$ ) solar cell to later records like the $>9 \%$-efficient poly[4,8-bis[(2-ethylhexyl) oxy]benzo[1,2-b:4,5-b']dithiophene-2,6-diyl][3-fluoro-2[(2-ethylhexyl)carbonyl]thieno[3,4-b]thiophenediyl]]: [6,6]phenyl $\mathrm{C}_{71}$-butyric acid methyl ester (PTB7: $\mathrm{PC}_{71} \mathrm{BM}$ ) [2] solar cells is certainly the optimization of energy levels of donor and acceptor molecules. As long as these energy levels are not optimized, substantial efficiency gains are possible by reducing the band gap to move closer to the optimum range of band gaps that give the best compromise between absorption and open-circuit voltage and by optimizing the energy offsets at the donor-acceptor heterointerface. This improvement of energetics was driven by the efforts of many synthetic chemists who were able to synthesize mostly new polymers [3-7] but also new fullerenes [8-10] or other small-molecule acceptors [11-14] to achieve higher photocurrents and opencircuit voltages. These optimization principles were discussed in many publications [15-18], among them, for instance, the work of Scharber et al. [19] in 2006, where the achievable efficiency was estimated as a function of energy levels based on empirical estimates of the maximum fill factor and quantum efficiency of the device. The efficiency development from MDMO-PPV via polymers like poly(3-hexylthiophene) (P3HT) or poly [N-9 "-hepta-decanyl-2,7-carbazole-alt-5,5(4',7'-di-2-thienyl-2', 1',3'-benzothiadiazole)] (PCDTBT)

*t.kirchartz@fz-juelich.de towards PTB7 can be understood from the optimization of energy levels as suggested by Scharber et al. However, the efficiency in the Scharber plot peaks at about $10 \%$ with no possibility for further improvement if none of the basic assumptions are changed. It is, therefore, obvious that improvements beyond the $10 \%$ predicted by Scharber have to tackle the assumptions of $65 \%$ for the fill factor (FF) and the external quantum efficiency (EQE). These assumptions are based on state-of-the-art results from the time around 2006 when the Scharber paper was published, but they represent by no means the theoretical maximum of what is achievable for these quantities. In principle, the external quantum efficiency can approach $100 \%$ in good inorganic solar cells, and there are no fundamental reasons that forbid $\mathrm{EQE}>90 \%$ in organic devices. The fill factor cannot be $100 \%$, but it is limited to the mid- to high 80s depending on the exact value of the open-circuit voltage and assuming voltage-independent charge collection and an ideality factor of 1 [20]. Thus, there is still a lot of room beyond the assumptions of Scharber et al. and further development of organic solar-cell efficiencies will have to target the physical mechanisms that allow highest FFs and better EQEs.

In this context, there are two aspects in particular that have to be tackled by organic solar-cell research. The first aspect is the empirical correlation between the maximum external quantum efficiency observed in experiment and the voltage loss $E_{\text {opt }} / q-V_{\mathrm{OC}}$ between the measured open-circuit voltage $V_{\mathrm{OC}}$ and the absorption onset $E_{\mathrm{opt}} / q$ of the polymer ( $q$ is the elementary charge). This empirical correlation as described by Li et al. [18] shows that the EQEs decrease towards lower voltage losses, which probably represents a loss due to geminate recombination and incomplete exciton dissociation 
at interfaces where the offsets are not big enough. This aspect is, of course, still linked to the question of how to optimize the energy levels at the donor-acceptor interface. The second aspect is how to optimize charge collection and minimize nongeminate recombination to allow high FFs at active-layer thicknesses that are sufficiently large in order to absorb nearly all of the above band-gap photons leading to an overall increase in the EQE.

Most high-efficiency polymers until relatively recently had optimum efficiencies at thicknesses around $100 \mathrm{~nm}$. This typically corresponds roughly to the first interference maximum, i.e., the lowest thickness that leads to a peak in the number of absorbed photons as a function of thickness $[21,22]$. Further increases in thickness will initially be detrimental for performance because the interferences lead to reduced absorption before the second interference maximum is hit typically at thicknesses above $200 \mathrm{~nm}$ [22,23]. At these thicknesses, however, charge collection is often not good enough anymore to allow for high FFs, and total efficiencies are decreasing such that the first interference maximum defines the optimum thickness and the better absorption of slightly thicker layers cannot be exploited [24-27]. With the notable exception of P3HT: $\mathrm{PC}_{61} \mathrm{BM}$ [28], most polymer:fullerene blends follow this general trend. However, recently, new polymers have been synthesized [27,29-36] that allow high FFs at thicknesses around the second interference maximum, thereby containing some of the main ingredients to overcome Scharber et al. assumptions.

The factors affecting charge collection and the FF include mobilities $\mu$, charge-carrier lifetimes $\tau$ or recombination constants $k$, as well as the effect of an inhomogeneous electric field caused, e.g., by doping or asymmetric mobilities [37]. There are many techniques to measure charge transport, recombination, doping, and space charge in organic or other solar cells that can be used to better understand the factors contributing to high FFs. However, it will be highly useful to be able to simply use the measured device parameters (FF, $V_{\mathrm{OC}}$, and short-circuit current density $J_{\mathrm{SC}}$ ), relate them to the absorber thickness, and use this as a figure of merit for charge collection. It is clear that the fill factor is highly sensitive to the voltage dependence and overall efficiency of charge collection, but because charge collection also depends strongly on device thickness and less strongly on light intensity and the recombination mechanism, it is not straightforward how to compare different FFs measured at different thicknesses or illumination intensities. Bartesaghi et al. [38] have suggested a figure of merit that correlates with the FF. However, there is still a substantial spread of FFs for a given value of the figure of merit making it difficult to directly correlate the measured FF with, e.g., the mobility-lifetime product of the device or with a similar parameter that captures the efficiency of charge collection relative to the efficiency of recombination in the device.
The present manuscript, therefore, intends to go one step further by refining and remodeling the proposed figure of merit and extending the scope of the study to different recombination mechanisms as well as nonbeneficial but realistic device configurations. This analysis clarifies the model's assumptions and shows in how far they are critical. The resulting limits are also valid for the study of Bartesaghi et al., which lacks a detailed analysis of the validity range of the proposed model. Our analysis is based on onedimensional drift-diffusion simulations of an effective medium which yields good results for many organic solar cells in which geminate recombination is negligible [39-42]. Eventually, the developed models enable the quantitative assay of the active layer's electronic quality by simple means under certain circumstances that are discussed in detail. We investigate the two limiting situations of free-to-free recombination (direct) and Shockley-Read-Hall recombination via a midgap trap, both of which have been reported to occur in organic solar cells [43-45]. While it is relatively easy to predict the fill factor from information of mobility, recombination rate, and thickness as long as the electric field is distributed homogeneously over the device thickness, major deviations occur when space-charge effects matter, i.e., when doping densities cannot be neglected or when asymmetric mobilities lead to the buildup of space charge under illumination. In these situations, the correlation between the fill factor and any figure of merit based on mobilities and lifetimes or recombination coefficients will break down. However, it is still possible to detect these situations from thicknessdependent device data and thereby avoid erroneous interpretations. Just like space-charge regions, an external series resistance can under certain circumstances affect the thickness dependence of the FF and can, thus, be detected. Since all these considerations - along with the presented figures of merit-require only the knowledge about a few essential device parameters (namely, $\mathrm{FF}, V_{\mathrm{OC}}, J_{\mathrm{SC}}$, and thickness), our study provides a valuable tool for experimentalists to estimate the influence of critical device properties such as contact resistance and electric field distribution on the FF and to estimate in how far they limit the device performance. Because the four required parameters $\left(\mathrm{FF}, V_{\mathrm{OC}}, J_{\mathrm{SC}}\right.$, and thickness) are commonly reported in the literature, it is even possible to apply our hypothesis retrospectively to data from a huge number of publications on organic solar-cell devices.

This allows us to come back to the original question of the development of organic solar-cell efficiencies over the past decade and show that this development was, until recently, mostly based on an improvement in energy levels rather than on a continuous reduction of nongeminate recombination causing improved charge collection.

\section{SIMULATIONS}

In an approach similar to Bartesaghi et al. [38], our study is based on drift-diffusion simulations carried out over a wide range of parameters typical for many organic solar 
cells. Huge data sets of $10^{4}$ points are created, and the behavior of the ensemble is analyzed. For each device simulation, the value of each parameter is chosen at random within its respective range specified in Table I. In an effective-medium approach, spatially independent properties are assigned to the photoactive material blend, which is, thus, described as a homogeneous medium. The energy difference between the acceptor lowest unoccupied molecular orbital (LUMO) and donor highest occupied molecular orbital (HOMO) is taken as the effective band gap [39-41,46-49]. By condensing microstructural effects into device parameters-such as mobility $\mu$ and direct recombination coefficient $k$-effective-medium simulations were successfully performed in many studies [39-42]. The free-charge-carrier generation rate $G$ is assumed to be constant across the entire active layer. To prevent unrealistically high short-circuit currents for thick devices, the generation rate is adjusted for different activelayer thicknesses and interference caused by flat interfaces is taken into account as shown in Fig. S1 [50-52]. Since voltage-dependent geminate recombination (recombination of excitons) is negligible for many of today's organic solar cells $[31,34,53-58]$, it is not considered here. Note that geminate recombination, which is independent of the applied electric field, merely reduces the generation rate of free carriers $G$, which does not affect the extraction and recombination dynamics of free carriers as long as chargecarrier concentrations are not changed drastically. The analysis is carried out for both direct (bimolecular, bandto-band, or free-to-free) and trap-assisted [monomolecular, Shockley-Read-Hall (SRH)] free-charge-carrier recombination models. For the sake of clarity and to compare with the results of Bartesaghi et al., we focus on the discussion of the direct recombination studies in the main text while the - in most cases similar - results of a trap-assisted recombination model are given in the Supplemental Material [52]. The range of the direct recombination coefficient $k$ or Shockley-Read-Hall lifetime $\tau$ is taken from comprehensive studies of various polymer:fullerene blends [59-61]. Direct recombination in an organic bulk heterojunction is often described to follow Langevin kinetics, where the mobility determines the recombination coefficient [38,54]. For many polymer blends, a mostly empirical and blend-specific Langevin-reduction factor is required to reproduce experimentally determined recombination coefficients [38,54,62], whereby the Langevin expression becomes merely an upper limit for direct recombination. In this work, the coefficient of direct recombination is randomly chosen from a wide parameter range, which automatically covers the case of reduced Langevin recombination. The properties of interlayers and metallic contacts are implemented via energetic barriers at the contacts, surface-recombination velocities, and an external series resistance.

We start our analysis by looking at undoped devices with balanced mobilities-implying the absence of spacecharge regions so that the active layer is fully depleted. There is no external contribution to the series resistance from the metallic contacts (note that organic solar cells use low-mobility materials so that the active layer contributes to the overall series resistance). Vanishing Schottky barriers $\varphi$ to the contacts and high and equal minority surfacerecombination velocities of $S=10^{7} \mathrm{~cm} \mathrm{~s}^{-1}$ are assumed (see the Supplemental Material [52] Fig. S2 for a representative band diagram). Deviations from this standard case are denoted in the text. Note that in the whole discussion, there is no preferential cell orientation related to the direction of light incidence on the device. Because of the spatially homogeneous generation rate, this orientation-independence also

TABLE I. Parameter ranges used in the simulations.

\begin{tabular}{lcccccc}
\hline \hline Parameter & & Figures 2 and S4 & Figure 3 & Figure 4 & Figure 5 & Unit \\
\hline Thickness & $d$ & $50-300$ & 100 & 100 & See figure & $\mathrm{nm}$ \\
Effective band gap & $E_{g}$ & $0.8-1.3$ & 1 & 1 & 1 & $\mathrm{eV}$ \\
Mobility & $\mu_{e, h}$ & $10^{-6}-10^{-2}$ & $10^{-6}-10^{-2}$ & $2 \times 10^{-3}$ & See figure & $\mathrm{cm}^{2}(\mathrm{~V} \mathrm{~s})^{-1}$ \\
Direct recombination coefficient & $k$ & $10^{-10}-10^{-13}$ & $5 \times 10^{-13}$ & $5 \times 10^{-13}$ & $10^{-12}$ & $\mathrm{~cm}^{-3} \mathrm{~s}$ \\
SRH lifetime & $\tau$ & $10^{-6}-10^{-4}$ & Not applicable & Not applicable & Not applicable & $\mathrm{s}$ \\
Illumination & $G$ & $G(d)$ & $G(d)$ & $G(d)$ & $G(d)$ & $\mathrm{cm}^{-3} \mathrm{~s}^{-1}$ \\
Doping & $N_{d}$ & 0 & 0 & 0 & See figure & $\mathrm{cm}^{-3}$ \\
External series resistance & $R_{s}$ & 0 & $0,3,5$ & 0 & 0 & $\Omega \mathrm{cm}^{2}$ \\
Schottky barriers & $\varphi$ & 0 & 0 & 0 & 0 & $\mathrm{eV}^{-3}$ \\
Minority surface recombination velocity & $S_{\text {min }}$ & $10^{7}$ & $10^{7}$ & $10^{-1}-10^{4}$ & $10^{7}$ & $\mathrm{~cm}^{-3} \mathrm{~s}^{-1}$ \\
Majority surface recombination velocity & $S_{\text {maj }}$ & $10^{9}$ & $10^{9}$ & $10^{9}$ & $10^{9}$ & $\mathrm{~cm}^{-3} \mathrm{~s}^{-1}$ \\
Dielectric constant & $\varepsilon_{r}$ & 3.5 & 3.5 & 3.5 & 3.5 & $10^{19}$ \\
Effective DOS & $N_{c, v}$ & $10^{19}$ & $10^{19}$ & $10^{19}$ & $\mathrm{~cm}^{-3}$ \\
\hline \hline
\end{tabular}


holds in the presence of a space-charge region at one of the contacts.

\section{BACKGROUND: FILL FACTOR OF ORGANIC SOLAR CELLS}

Even for the simple case of a homogeneously distributed electrical field, there is no simple relation between fill factor and thickness evident from the simulations presented in Fig. 1. As mentioned previously, the complex behavior stems from the multitude of parameters that influence the voltage-dependent competition between charge-carrier extraction and recombination, which eventually governs the fill factor of a device. In particular, the qualitative behavior depends on the mobility, which is varied for the different curves in Fig. 1, as well as the recombination coefficient, which is kept constant in this example. As a consequence, the interpretation of the experimental thickness series in terms of fill factor is cumbersome and rarely reported [37], although thickness series are typically prepared during device optimization.

By means of drift-diffusion simulations, Bartesaghi et al. recently showed that the fill factor qualitatively follows the logarithm of the parameter

$$
\theta_{\mathrm{dir}}=\frac{V_{\mathrm{int}}^{2}}{d^{4} G} \frac{\mu_{e} \mu_{h}}{k}
$$

which is derived from analytical considerations of the extraction and recombination dynamics in an organic solar cell at short-circuit conditions [38]. In Eq. (1), $\mu_{e}$ and $\mu_{h}$ are the electron and hole mobilities, respectively, $k$ is the coefficient of direct recombination, $G$ is the generation rate, and $d$ is the thickness of the photoactive layer. The internal voltage $V_{\text {int }}$ across the active layer is defined in Ref. [38] by $q V_{\text {int }}=E_{\mathrm{LUMO}}-E_{\mathrm{HOMO}}-0.4 \mathrm{eV}$ and results from the

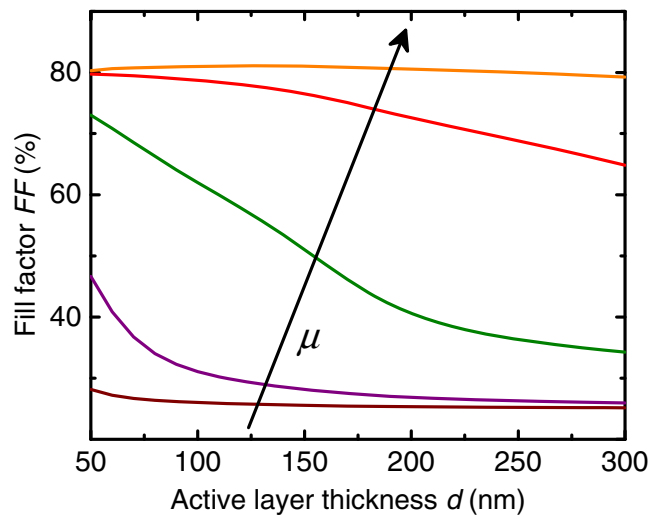

FIG. 1. Simulated device fill factors of typical organic bulk heterojunction with active-layer thicknesses between 50 and $300 \mathrm{~nm}$. All other simulation parameters remain unchanged along one curve. No characteristic relation between fill factor and thickness can be identified, and the behavior changes qualitatively for typical mobilities $\mu$ between $10^{-6}$ and $10^{-2} \mathrm{~cm}^{2} /(\mathrm{V} \mathrm{s})^{-1}$. energetic difference of the acceptor's LUMO and the donor's HOMO with a correction term that accounts for band bending. Note that Bartesaghi et al. used the reciprocal expression of Eq. (1) in their work. For clarity, we refer to parameters such as $\theta$ that describe the ratio of extraction and recombination in an organic solar cell as the collection coefficient.

Although this analytical treatment is certainly valuable for its theoretical foundation and qualitative predictions, it is based on assumptions that are not fully valid for the discussed conditions. Specifically, the expression is derived at short circuit where the electric field which drives charges to the electrodes is considerably larger than at the maximum power point which is typically closer to open-circuit than short-circuit conditions (in a very recent report, Neher et al. [63] derived another figure of merit that does not depend on the applied voltage). Also, Eq. (1) predicts equally weighted exponents for the slow- and fast-carrier species, which is in contrast to experimental observations that found the mobility of the slower charge-carrier species to be decisive for transport [43,64-66], as we discuss later. Such shortcomings are likely to explain the large scatter in the presented data and raise the question about the relative importance of the quantities that determine the FF. There are, for example, analytical estimates for the efficiency of charge collection (such as the model by Crandall $[67,68]$ ), where the mobility and lifetime always appear together as the $\mu \tau$ product. Thus, one expects that, for instance, in a model based on SRH recombination, mobility and lifetime are equally important for the FF. However, this is, in practice, not the case, because while it may indeed be only the $\mu \tau$ product that controls charge collection, the FF relates the efficiency of charge collection at the maximum power point to the product of $J_{\mathrm{SC}}$ and $V_{\mathrm{OC}} \cdot V_{\mathrm{OC}}$, for instance, increases with lifetime but often decreases with mobility [48,69-71]. Thus, one expects that for the $\mathrm{FF}=\left[J_{\mathrm{MPP}} V_{\mathrm{MPP}} /\left(J_{\mathrm{SC}} V_{\mathrm{OC}}\right)\right]$, the mobility is more important than the lifetime which increases both the numerator and the denominator of the FF equation. A model taking the relative importance of different parameters into account should, therefore, reduce the spread of data points.

This somewhat more heuristic approach can overcome the various discussed shortcomings of the analytical treatment and lead to a more precise and practical description, as we discuss in the remainder of this paper.

\section{RESULTS AND DISCUSSION}

\section{A. Model}

In order to obtain a model compatible with experimental work that can also be applied to the reported data from the literature, the internal voltage in Eq. (1) is replaced with the open-circuit voltage $V_{\mathrm{OC}}$, and the generation rate is substituted by the short-circuit current density $J_{\mathrm{SC}}$ via $q d G=J_{\mathrm{SC}}$, which holds for the case of complete 
collection of all generated charges. In order to compare their experimental data to the simulation, Bartesaghi et al. used the same substitutions when the polymer's HOMO and LUMO or the generation rate was unknown. Both $V_{\mathrm{OC}}$ and $J_{\mathrm{SC}}$ are obtained in $J-V$ measurements which are almost always carried out when solar-cell devices are prepared. For the case of balanced mobilities, $\mu_{\mathrm{e}}=\mu_{\mathrm{h}}:=\mu$, the modified analytical expression takes the form

$$
\theta_{\mathrm{dir}}^{\prime}=\frac{V_{\mathrm{OC}}^{2}}{d^{3} J_{\mathrm{SC}}} \frac{\mu^{2}}{k}
$$

The relation between the fill factor and $\theta_{\text {dir }}^{\prime}$ is depicted in Fig. 2(a) where the coordinates of each data point are obtained through the extraction of FF, open-circuit voltage $V_{\mathrm{OC}}$, and short-circuit current density $J_{\mathrm{SC}}$ from the computed $J-V$ curves, while mobility $\mu$, recombination coefficient $k$, and thickness $d$ are inputs to the simulations. Although the overall trend of large $\theta_{\text {dir }}^{\prime}$ leading to large fill factors is evident from Fig. 2(a), the data are considerably scattered in the linear part of the distribution. As can be seen from the Supplemental Material [52] Fig. S3(a), this scatter does not originate from the introduction of $V_{\mathrm{OC}}$ and $J_{\mathrm{SC}}$ to the collection coefficient as defined by Bartesaghi et al. in Eq. (1) where internal voltage $V_{\text {int }}$ and generation rate $G$ are used instead. For the case of unbalanced charge transport-shown in the publication of Bartesaghi et al. and discussed later in this manuscript-the scatter becomes much stronger.

In a next step, the scatter is reduced by introducing a normalized fill factor $\mathrm{FF}_{n}$ and varying the exponents in Eq. (2) to find the relative weight of the constituting quantities and attain an optimized collection coefficient $\gamma_{\text {dir }}$. The normalization of the fill factor accounts for the dependence of the maximum fill factor $\mathrm{FF}_{\max }$ on the opencircuit voltage $V_{\mathrm{OC}}$, which results from maximizing the fillfactor definition $\mathrm{FF}[V, J(V)]$ using a one-diode model for the current-voltage characteristic $J(V)$. Several analytical functions which describe the solution of the problem exactly or approximately with varying accuracy were discussed by Green [72,73]. Following Green's earlier work [73], we choose the simple relation

$$
\mathrm{FF}_{\text {max }}=\frac{a V_{\mathrm{OC}}}{V_{\mathrm{OC}}+b}
$$

and set the lower limit of possible fill factors to

$$
\mathrm{FF}_{\min }=25 \%
$$

corresponding to a nonrectifying device with a linear $J-V$ curve. Subsequently, we normalize the fill factor via

$$
\mathrm{FF}_{n}=2 \frac{\mathrm{FF}-\mathrm{FF}_{\min }}{\mathrm{FF}_{\text {max }}-\mathrm{FF}_{\min }}-1
$$
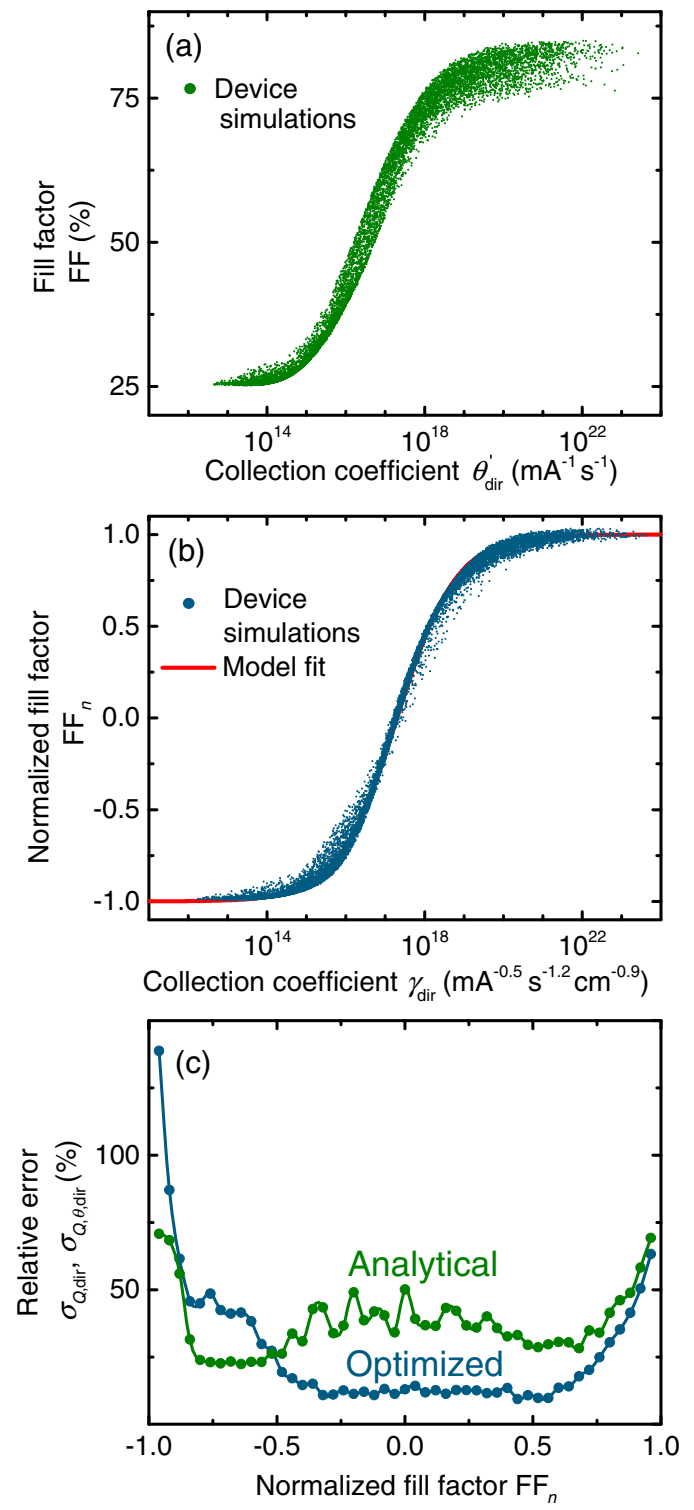

FIG. 2. Dependence of the FF on two differently defined collection coefficients $\theta_{\text {dir }}^{\prime}$ and $\gamma_{\text {dir }}$ that reflect the competition of charge-carrier extraction and recombination in organic solar cells. Each of the $10^{4}$ data points in (a) and (b) corresponds to a device simulation for the case of direct recombination and balanced mobilities with input parameters chosen at random from the intervals given in Table I. (a) Analytical considerations of Bartesaghi et al. [Eq. (2), Ref. [38] ] yield $\theta_{\text {dir }}^{\prime}$ (b) Optimizing the relative weight of the quantities that constitute the collection coefficient $\gamma_{\text {dir }}$ [Eq. (7a)] and normalizing the FF [Eqs. (3)-(5)] result in a significant reduction of the scatter in the data. The error [Eq. (10)] of extracting the here-defined electronic quality factor $Q$ [Eq. (8)] through Eq. (9) is shown in (c) for both models over several intervals of $\mathrm{FF}_{n}$ which quantifies the enhanced precision of the optimized model.

which maps the FF onto the range $(-1,1)$. The constants $a$ and $b$ in Eq. (3), along with the optimized exponents of the collection coefficient $\gamma_{\text {dir }}$ are found by fitting the data pairs of the normalized fill factor $\mathrm{FF}_{n}$ collection coefficient $\gamma_{\text {dir }}$ to the fit function 


$$
\mathrm{FF}_{n, \text { fit }}\left(\gamma_{\text {dir }}\right)=\tanh \left\{\alpha_{\text {dir }} \ln \left(\gamma_{\text {dir }} / \gamma_{0, \text { dir }}\right)\right\},
$$

where $\alpha_{\text {dir }}$ and $\gamma_{0 \text {,dir }}$ are fit parameters as well. The function fulfills the requirements of a monotonous and bounded function that produces the $\mathrm{S}$ shape discernable in Fig. 2(a) and shares the range $(-1,1)$ of $\mathrm{FF}_{n}$. The collection coefficient is found to be

$$
\gamma_{\mathrm{dir}}=\frac{V_{\mathrm{OC}}^{2.0}}{d^{3.5} J_{\mathrm{SC}}^{0.5}} \frac{\mu^{2}}{k^{0.8}} .
$$

Comparing Figs. 2(b) and 2(a) shows that the introduction of the normalized fill factor $\mathrm{FF}_{n}$ reduces the scatter at high FFs close to $\mathrm{FF}_{\max }$ where the FF is bound only by the fundamental dependence on $V_{\mathrm{OC}}$ [Eq. (3)]. The optimized exponents of the collection coefficient $\gamma_{\text {dir }}$ substantially narrow the linear part of the distribution. Note that the density of points that deviate the most from the fit function [red curve in Fig. 2(b)] is much lower than the point density along the curve. The improved precision of the optimized collection coefficient is quantified in the next section.

For the case of trap-assisted SRH recombination, where the lifetime $\tau$ replaces the direct recombination coefficient, the optimization procedure yields

$$
\gamma_{\mathrm{SRH}}=\frac{V_{\mathrm{OC}}^{2.0}}{d^{4.3}} \mu^{2} \tau^{1.6} .
$$

The fit parameters $a, b, \gamma_{0}$, and $\alpha$ are given in Table II for both recombination models. Equations (7a) and (7b) use the units given in Table I, except for the device thickness $d$, which needs to be entered in centimeters. $V_{\mathrm{OC}}$ is in volts, $J_{\mathrm{SC}}$ in $\mathrm{mA} \mathrm{cm}^{-2}$, and FF in percent. Note that both collection coefficients are not dimensionless. The results differ for exceptionally strong or weak recombination shown in the Supplemental Material [52] Figs. S4(b) and S4(c), which is, however, uncommon for organic solar cells.

Although the models for direct and trap-assisted recombination represent limiting cases, they produce similar results. The obtained expressions for both recombination types show minor deviations from the theoretical models for $p-i-n$ solar cells of Crandall [67] and Hecht [74] for trap-assisted recombination (note the resemblance to the

TABLE II. Fit parameter values for direct and Shockley-ReadHall recombination models.

\begin{tabular}{lcccc}
\hline \hline & $a$ & $b$ & $\gamma_{0}$ & $\alpha$ \\
\hline Direct & 0.92 & 0.09 & $2 \times 10^{17}$ & 0.29 \\
& & & $\mathrm{~mA}^{-0.5} \mathrm{~s}^{-1.2} \mathrm{~cm}^{-0.9}$ & \\
SRH & 0.92 & 0.09 & $1.3 \times 10^{4}$ & 0.19 \\
& & & $\mathrm{~mA}^{-0.5} \mathrm{~s}^{-1.2} \mathrm{~cm}^{-0.9}$ & \\
\hline \hline
\end{tabular}

ratio of drift length to thickness) and Bartesaghi et al. [38] for direct-reduced Langevin recombination. In particular, the exponents of the quantities that describe recombination are slightly lower than in the analytical expressions $\left[\mu^{2} / k\right.$ for direct recombination and $(\mu \tau)^{2}$ for Shockley-Read-Hall recombination]. The deviations can be understood from the differences between the approaches: while the analytical models describe the efficiency of charge-carrier collection, our collection coefficient is optimized to precisely predict the fill factor. Lower recombination improves charge collection at the maximum power point and, at the same time, increases $V_{\mathrm{OC}}$. Thereby, the numerator and the denominator of the fill-factor definition are increased $\mathrm{FF}=\left[J_{\mathrm{MPP}} V_{\mathrm{MPP}} /\left(J_{\mathrm{SC}} V_{\mathrm{OC}}\right)\right]$, which leads to a decreased weight of recombination. The thickness dependence is almost the same in Eqs. (1) and (7a) if the additional thickness dependence of the short-circuit current is taken into account in the case of direct recombination. Since Shockley-Read-Hall recombination is linear with excess charge-carrier concentration, it is (nearly) independent of the generation rate and, thus, the short-circuit current.

The weights of the constituting quantities in Eqs. (7a) and (7b) are optimized relative to the weight of the mobility whose exponent is fixed at 2 . The fitting procedure yields stable results up to the given digit and is particularly sensitive to the weight of the recombination coefficient, while the relative weight of $J_{\mathrm{SC}}$ and $d$ is less critical since both quantities are related through the generation rate.

\section{B. Electronic quality factor and model precision}

A crucial factor in the collection coefficient in Eq. (7a) is the ratio of mobility $\mu$ and recombination coefficient $k$-both properties of the material blend composing the active layer-which defines an electronic quality factor

$$
Q_{\text {dir }}=\frac{\mu^{2}}{k^{0.8}} .
$$

In the case of trap-assisted recombination, the electronic quality factor is defined by

$$
Q_{\mathrm{SRH}}=\mu^{2} \tau^{1.6} .
$$

By substituting the collection coefficient [Eq. (7a)] into the fit function $\left[\mathrm{FF}_{n, \text { fit }}\right.$, Eq. (6)], setting $\mathrm{FF}_{n, \text { fit }}=\mathrm{FF}_{n}$ and solving for $Q_{\mathrm{dir}}$, the electronic quality factor can be estimated accurately from basic device characterization and our fit model via

$$
Q_{\text {fit,dir }}=\gamma_{0, \mathrm{dir}} \frac{d^{3.5} J_{\mathrm{SC}}^{0.5}}{V_{\mathrm{OC}}^{2}} \exp \left(\frac{\operatorname{arctanh} \mathrm{FF}_{n}}{\alpha_{\mathrm{dir}}}\right) .
$$

Only the measurement of the active-layer thickness $d$ and the current-voltage curve yielding $V_{\mathrm{OC}}, J_{\mathrm{SC}}$, and FF is required. This is remarkable because the mobility and 
recombination coefficient are difficult to obtain individually in experiments and values vary for different methods $[68,75,76]$.

The error on the electronic quality factor $Q_{\text {fit,dir }}$ predicted from our model can be evaluated from the simulation results by comparing it to the actual value of $Q$ defined by Eq. (8a) which results from simulation inputs. We define the error

$$
\sigma_{Q, \mathrm{dir}}=\frac{\left|Q_{\mathrm{dir}}-Q_{\mathrm{fit}, \mathrm{dir}}\right|}{Q_{\mathrm{dir}}}
$$

The collection coefficient based on the model of Bartesaghi et al. [Eq. (1)] defines a different electronic quality factor $Q_{\theta, \text { dir }}=\mu^{2} / k$. Following the previous procedure of introducing a normalized fill factor and fit function [see the Supplemental Material [52] Fig. S3(b)], the model's estimated electronic quality factor $Q_{\mathrm{fit}, \theta \text {,dir }}$ and its error $\sigma_{Q, \theta, \text { dir }}$ can be evaluated and compared to our model. The two regions with very low and very high normalized fill factor $\mathrm{FF}_{n}$ in Fig. 2(c) are difficult to interpret in terms of electronic quality, as indicated by the large error of the estimated quality factor for both models. Huge changes in mobility and lifetime lead only to moderate changes in FF if the FF is already nearly ideal or if it is already close to $25 \%$. However, at intermediate FFs, the enhanced precision of our collection coefficient $\gamma_{\text {dir }}$ is obvious. In this regime, changes in the mobility and recombination constant (or lifetime) directly translate into changes in $\mathrm{FF}_{n}$, which allows the extraction of the electronic quality factor $Q_{\text {dir }}$.

Figure 3(a) compares our model to two thickness series from the literature [30] and DT-PDPP2-TT: $\mathrm{PC}_{71} \mathrm{BM}$ [31] that reached high FFs at thicknesses around the second absorption maximum. One data point is used to extract $Q_{\text {dir }}$, which is then assumed to be constant for all thicknesses of the series. Both series agree well with the model except for the highest values of $\gamma$ which correspond to the devices with the lowest absorber thickness in the series. This behavior is typical because at high FF and $\gamma$ the external series resistance becomes a limiting factor of the FF, as we discuss in the next section. Additionally, for low device thicknesses, often shunts become more relevant for the FF, and, thus, the FF stays short of its potential if only the efficiency of charge collection is taken into account.

\section{Contacts}

For good bulk properties indicated by large collection coefficients $\gamma$ and high fill factors, the influence of imperfect contacts becomes limiting. More specifically, the highest obtainable fill factor decreases. In this context, the term "contact" does not exclusively refer to the metallic connection to the external circuit but also includes properties coming from interlayers. For example, in terms of device modeling, the high-charge selectivity of a good electron-blocking hole-transport layer can be represented by a high-surface-recombination velocity for holes (majority carriers, $S_{\text {maj }}$ ) and a low-surface-recombination velocity for electrons (minority carriers, $S_{\min }$ ) instead of an additional layer in the simulated stack. Interlayers whose energy levels are well matched to the active layer mean a low Schottky barrier $\varphi$ used in simulations. Finally, since interlayers are nonideal conductors, they will also contribute to the external series resistance $R_{s}$, which lowers the fill factor.

Figure 3(b) shows the normalized fill factor $\mathrm{FF}_{n}$ for a set of curves with varying external series resistance. While the linear regime is barely influenced (also compare the Supplemental Material [52] Fig. S5), the curves saturate at lower levels for increasing series resistance. It is noteworthy that the original curve without external resistance can be regained for a known resistance [dashed line in Fig. 3(b)] by adapting the maximum fill-factor definition from Eq. (3) to [77]

$$
\mathrm{FF}_{\max , R_{s}}=\mathrm{FF}_{\max }\left(1-\frac{R_{s} J_{\mathrm{SC}}}{V_{\mathrm{OC}}}\right) .
$$

A Schottky barrier between the active layer and contacts reduces the built-in voltage which impedes the extraction of charge carriers and deteriorates the fill factor [78].

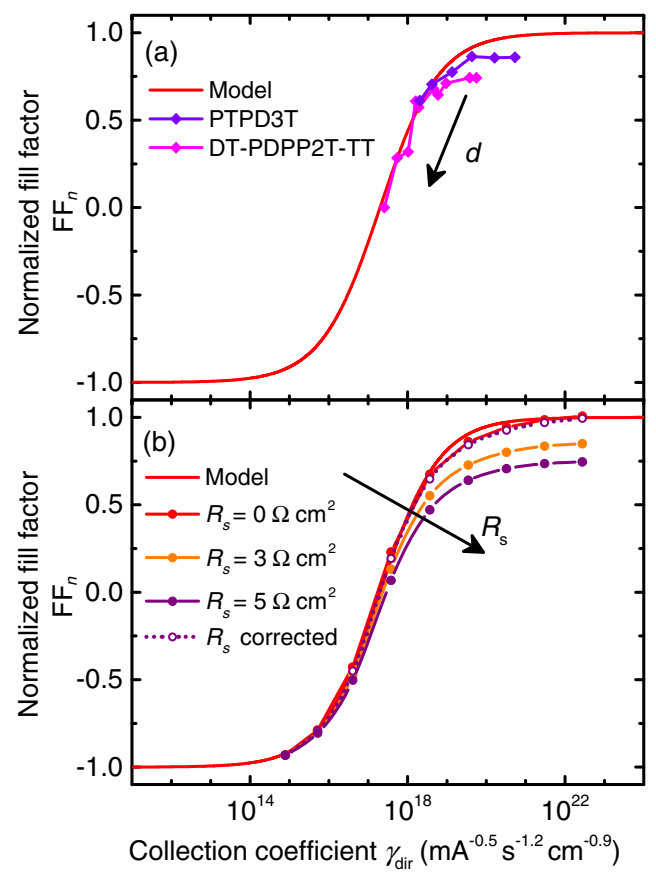

FIG. 3. (a) Literature data on solar cells with varying activelayer thickness made from the polymers PTPD3T [30] and DT-PDPP2T-TT [31] are in good agreement with our model. Thin devices show FF values that are below the model's prediction which can be explained with finite external series resistances as shown in (b). For known resistance, the data can be corrected by Eq. (11) as shown for the case of a $5-\Omega \mathrm{cm}^{2}$ series resistance. 
It is well known that higher values of the open-circuit voltage $V_{\mathrm{OC}}$ can be achieved in different types of solar cells by reducing the surface-recombination velocity of minority charge carriers [79] and many improvements in standard $c$-Si technology are based on this fact [80]. Higher $V_{\mathrm{OC}} \mathrm{S}$ then allow higher FFs as captured in Eq. (3). We focus on the impact of the surface-recombination velocity $S_{\min }=: S$ of minority carriers and assume that the majority carriers are injected and extracted fast enough [78]. For solar cells from low-mobility materials, effects related to surface recombination become visible only if the bulk properties are good, referring to a range of high values of the collection coefficient $\gamma$ and normalized fill factor $\mathrm{FF}_{n}$. Devices with lower $\gamma$ are limited by poor bulk properties, just as for the external series resistance discussed above. Figure 4(a) depicts the simulated $J-V$ curves of two high- $\gamma$ devices with different surface-recombination velocities $S$.

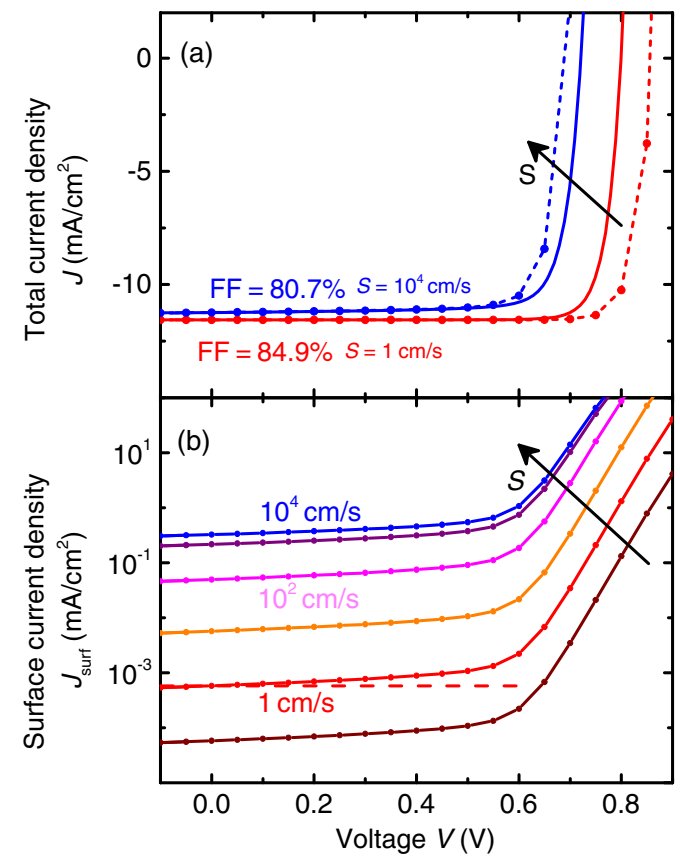

FIG. 4. (a) Solid lines show the simulated $J-V$ characteristic of a device with high collection coefficient $\gamma_{\text {dir }}$ for different values of surface recombination $S$ of minority carriers. Apart from the expected drop in $V_{\mathrm{OC}}$ for strong surface recombination (blue), a decrease in FF is observed which is caused by the slight voltage dependence of the $J$ - $V$ curve at low voltages and can be explained by the contribution of the surface-recombination current density $J_{\text {surf }}$ depicted in (b). The red curve in (b) where the dashed line indicates $J_{\text {surf }}$ at zero voltage illustrates the typical voltage dependence of $J_{\text {surf }}$. However, only for large enough values of $S, J_{\text {surf }}$ contributes significantly $\left(J_{\text {surf }} \gtrsim 10^{-2} \mathrm{~mA} / \mathrm{cm}^{2}\right)$ to the total current density. Consequently, the surface-recombination current density (shifted by $J_{\mathrm{SC}}$ ) indicated by the dashed lines in (a) reproduces the $J-V$ curve well in the case of strong surface recombination. For suppressed surface recombination (red), the surface contribution to the total current is negligible, and the $J-V$ curve is determined by the bulk recombination current.
Apart from the expected reduction in $V_{\mathrm{OC}}$ for larger $S$, there is a clear voltage dependence at low voltages which can be assigned to a voltage-dependent contribution of the surfacerecombination current. For an illuminated device, the total current is constituted by a generation current $J^{\text {gen }}$, a recombination current coming from the bulk $J_{\text {bulk }}^{\text {rec }}$, and one coming from the surfaces $J_{\text {surf }}^{\text {rec }}$ :

$$
J=J^{\mathrm{gen}}+J_{\text {bulk }}^{\mathrm{rec}}+J_{\text {surf }}^{\mathrm{rec}} .
$$

Since we consider a fully symmetric device with equal surface-recombination velocities of electrons and holes at the respective contact $S_{n}=S_{p}=S$, both surfaces contribute in the same way so that the recombination current from the surfaces is given by

$$
J_{\mathrm{surf}}^{\mathrm{rec}}=q S_{n} n(0)+q S_{p} p(d)=2 q S n(0),
$$

where the minority charge-carrier densities at the corresponding contact are denoted by $n(0)$ and $p(d)$. For an illuminated device shown in Fig. 4(b), there is an almost flat regime of the surface current at low voltages and a steeper one around $V_{\mathrm{OC}}$ which is responsible for changes in open-circuit voltage $V_{\mathrm{OC}}$ with surface-recombination velocity $S$. The inverse slope of the logarithm of the surface-recombination current vs $V_{\mathrm{OC}}$ is proportional to the diode's ideality factor. Around $V_{\mathrm{OC}}$, the ideality factor of the surface current is 1 and, therefore, equal to the bulk ideality factor for the case of direct recombination so that bulk and surface current have the same voltage dependence. Consequently, $S$ contributes to the diode saturation current $J_{0}$ and thereby $V_{\mathrm{OC}}$. This mechanism is covered by the fillfactor normalization in Eq. (3) and does not lead to a deviation from our model.

The additional dependence of the FF on the surfacerecombination velocity $S$ originates from the low-voltage regime where the voltage dependence is small, which implies a large ideality factor that is known to be detrimental for the FF [77]. However, this high-ideality contribution is relevant only for large $S$ because only then the surface-recombination current exceeds values of at least $10^{-2} \mathrm{~mA} \mathrm{~cm}^{-2}$ as can be seen from the logarithmic depiction of the surface-recombination current in Fig. 4(b). Only then, the surface-recombination current contribution to the total recombination current of approximately $12 \mathrm{~mA} \mathrm{~cm}^{-2}$ is significant. In that case, the surface-recombination current reproduces the $J-V$ curve very well, as seen from the dashed line in Fig. 4(a), while in the favorable case of low $S$, no significant contribution from the surface-recombination current is observed. This absence of voltage-dependent current at low voltages leads to an enhancement of the FF from $81 \%$ for high-surface-recombination velocities to $85 \%$ for low-surface-recombination velocities, which has not been recognized by Bartesaghi et al. who report no difference of the FF for infinite- and low- $\left(S=10^{-2} \mathrm{~cm} \mathrm{~s}^{-1}\right)$ 
surface-recombination velocity. In their study, differences in FFs are partly discarded by the large scatter at high FFs.

\section{Space charge}

The electric field within the active layer of organic bulk heterojunction solar cells is often assumed to be constant, meaning that the conduction and valence bands run linearly between the anode and cathode. Apart from band bending at the contacts, this is often a reasonable assumption. However, in the presence of sufficient space charge induced either by intentional or unintentional doping or by the presence of large concentrations of injected or photogenerated electrons and holes with asymmetric mobilities, the electric field is redistributed so that a field-free region with flat bands and a space-charge region with enhanced electric field form $[37,65,81-86]$. In organic solar cells, the spacecharge region forms at one of the organic semiconductormetal interfaces, as shown in the band diagram in the Supplemental Material [52] Fig. S2. Minority carriers generated in the flat-band region of a device need to diffuse to the space-charge region before the electrical field in the space-charge region separates them from the majority carriers so that they can be extracted at the contacts. While this process is efficient for high-mobility systems with long diffusion lengths such as crystalline silicon, it degrades the performance of low-mobility organic systems with short diffusion lengths, mainly because of a reduction in short-circuit current $J_{\mathrm{SC}}$ [37,81-83,86,87]. Devices based on polymers with improved electronic properties recently achieved high fill factors even for thick active layers [27,29-35]. This underlines the growing importance of space-charge effects because the width of the active layer and the space-charge region can be of the same order even for moderate doping levels or asymmetries in charge-carrier mobilities. Note that for a device series with increasing thickness-and, therefore, decreasing ratio between the width of the spacecharge region and the active layer-space-charge effects will first manifest themselves in a drop in $J_{\mathrm{SC}}$. By increasing the active-layer width further beyond the optimum efficiency, a deviation of the FF from our model enables first deductions on the presence of space charge by easy means, which can then be further characterized by the Suns- $J_{\mathrm{SC}}$ method or voltage-dependent capacitance measurements $[81,88,89]$.

Doping of a device (typically unintentional $p$-type doping [81,90-92]) leads to a space-charge region whose width decreases with increasing doping concentration. The impact on the fill factor can be seen from Fig. 5(a) showing simulations of a typical device with a doping level of $N_{d}=$ $5 \times 10^{16} \mathrm{~cm}^{-3}$ for thicknesses between $50 \mathrm{~nm}$ (highest $\gamma$ ) and $750 \mathrm{~nm}$ (lowest $\gamma$ ). The main difference between the model curves for doped and undoped devices is the saturation of the normalized fill factor $\mathrm{FF}_{n}$ for thick devices in the case of doped devices. In this regime, low mobilities and/or high recombination coefficients are predominant so that only carriers from a very narrow collection zone can diffuse to the space-charge region where they get separated. This process is independent of the applied voltage as soon as the space-charge region and the collection zone are smaller than the device thickness for all voltages. Thus, the presence of space charge leads to poor carrier collection for small mobility systems causing a low $J_{\mathrm{SC}}$ and a decrease in efficiency. However, the collection in the neutral zone does not depend on the applied voltage so that the FF decreases much more slowly for increasing thickness and decreasing collection coefficient $\gamma$. The saturation level depends on the doping level or asymmetry ratio of the mobilities discussed below and can explain the elevated FF of the 1000-nmthick device based on the polymer blend PBnDT FTAZ: $\mathrm{PC}_{61} \mathrm{BM}$ [34] [green diamonds in Fig. 5(a)] (the decrease in FF of the thinnest device with largest $\gamma$ is expected to be due to a reduced shunt resistance).

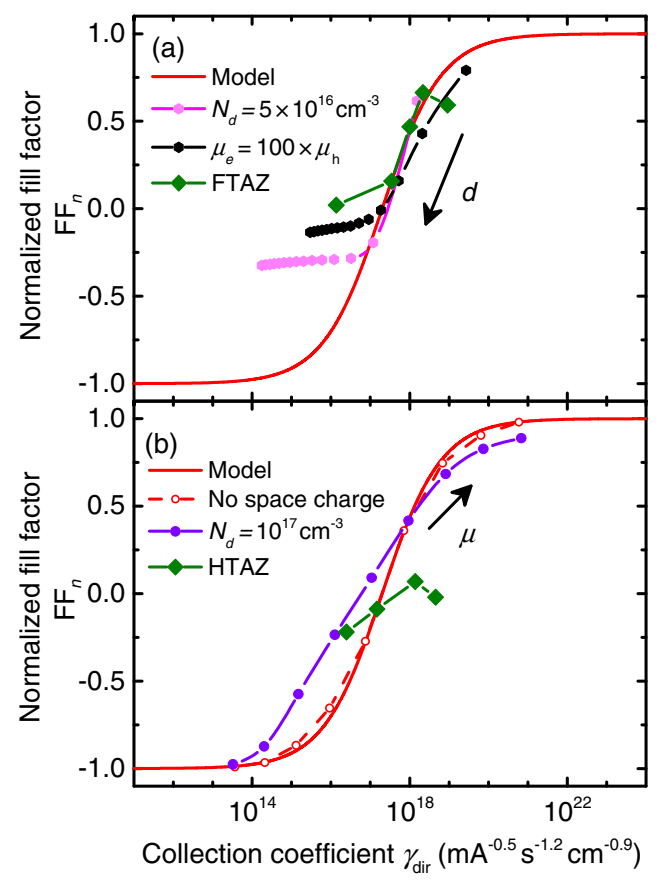

FIG. 5. The presence of a space-charge region caused by doping or asymmetric charge-carrier mobilities leads to inefficient carrier collection. Therefore, $J_{\mathrm{SC}}$ and PCE decrease while the FF increases at low collection coefficients $\gamma_{\text {dir }}$ as shown in (a) because charge-carrier collection becomes independent of voltage. The active-layer thickness along the simulated curves is increased from 50 to $750 \mathrm{~nm}$, while the mobility is kept constant [doped device $\mu=10^{-4} \mathrm{~cm}^{2}(\mathrm{Vs})^{-1}$, device with unbalanced mobility $\mu_{h}=10^{-5} \mathrm{~cm}^{2}(\mathrm{~V} \mathrm{~s})^{-1}$, and $\left.\mu_{e}=10^{-3} \mathrm{~cm}^{2}(\mathrm{~V} \mathrm{~s})^{-1}\right]$. The literature data (green) on the polymer FTAZ [34] show a similarly enhanced FF for the thickest device (1000 nm). For very high doping levels shown in (b), the slope between $\mathrm{FF}_{n}$ and $\gamma_{\text {dir }}$ changes which can explain the literature report on devices with thicknesses up to $750 \mathrm{~nm}$ based on the polymer HTAZ [34]. The simulated device has a thickness of $250 \mathrm{~nm}$ while the mobility is varied between $10^{-6}$ and $10^{-2} \mathrm{~cm}^{2}(\mathrm{~V} \mathrm{~s})^{-1}$. 
A space-charge region causing a similar saturation of the FF at large thickness results from unbalanced mobilities as shown in Fig. 5(a)[37,65,87]. In organic bulk heterojunctions, electrons are transported to the contacts via the acceptor material (usually a fullerene derivative) and holes via the donor material (polymer or small molecule). In addition, the blend morphology strongly affects the transport paths and amount of useable transport channels. Thus, differences in the mobilities between both phases of several orders of magnitude are quite common and cause an accumulation of charge at the contact that extracts the slow carrier species. The resulting space charge decreases the device efficiency via $J_{\mathrm{SC}}$ while causing a saturation of the fill factor at low $\gamma$ for the same reasons discussed above. Optimized fabrication steps such as annealing help to accomplish a material blend with balanced mobilities $[76,84,93]$.

Several studies suggest a dominant influence of the slower charge-carrier species for transport in organic solar cells $[43,64-66]$. This poses the question of the exact weight distribution between the slow and fast charge-carrier species in the collection coefficient $\gamma$ which is modified for the case of asymmetric mobilities by dividing the mobility into two separate factors. We find that putting a strong weight on the mobility of the slow carriers

$$
\mu^{2} \rightarrow \mu_{\text {slow }}^{1.6} \mu_{\text {fast }}^{0.4}
$$

yields the best overlap between the data with different asymmetry ratios. An effective mobility has been proposed that puts equal weight on the slow and fast charge-carrier species which is also predicted by the analytical treatment of Bartesaghi et al. [Eq. (1)]. While the definition of an effective mobility resulting from the presence of a fast and slow charge-carrier species has been proposed elsewhere [94], our results show the dominant role of the slow carrier species for transport as stated in the before-mentioned publications $[43,64-66]$. Contrary to this, the analytical treatment by Bartesaghi et al. [Eq. (1)] predicts equal weight of both charge carriers which adds to the enhanced scattering in their publication as shown in the Supplemental Material [52] Fig. S7. Our data suggest a dominant but not exclusive contribution of the slower charge-carrier species to charge transport.

Figure 5(b) shows that high doping concentrations $N_{d}=$ $10^{17} \mathrm{~cm}^{-3}$ change the slope of the normalized fill factor $\mathrm{FF}_{n}$ collection coefficient $\gamma$ relation. Here, a wide $\gamma$ range is covered by varying the mobility between $10^{-6} \mathrm{~cm}^{2}(\mathrm{~V} \mathrm{~s})^{-1}$ and $10^{-2} \mathrm{~cm}^{2}(\mathrm{~V} \mathrm{~s})^{-1}$ while the thickness is kept fixed at $200 \mathrm{~nm}$. The behavior reflects that of a large ensemble of devices given by clouds of points with different asymmetry ratios and doping levels shown in the Supplemental Material [52] Fig. S6 and is similar to experimental data for varying thicknesses of an active layer from a polymer blend of PBnDT-HTAZ: $\mathrm{PC}_{61} \mathrm{BM}$ [34].

\section{OSC EFFICIENCIES: HISTORY AND FUTURE}

In their paper of 2006, Scharber and co-workers [19] illustrated a simple guideline to boost power-conversion efficiencies of organic solar cells from P3HT's record at that time of $4.4 \%$ [28] up to around $10 \%$. Their work emphasized the large potential that was in the favorable arrangement of the polymer's HOMO and LUMO levels when PCBM is assumed as the acceptor material [19]. Scharber et al. use an empirical estimate for the opencircuit voltage $V_{\mathrm{OC}}$ given by the interfacial band gap minus an offset of $0.3 \mathrm{eV}$,

$$
q V_{\mathrm{OC}}=E_{\mathrm{LUMO}, \mathrm{PCBM}}-E_{\mathrm{HOMO}, \mathrm{pol}}-0.3 \mathrm{eV},
$$

which holds for many investigated organic solar cells $[18,19,95]$. In terms of the polymer band gap $E_{g, \text { pol }}$, this expression translates to

$q V_{\mathrm{OC}}=E_{g, \mathrm{pol}}-\Delta E_{\mathrm{LUMO}}-0.3 \mathrm{eV}=E_{g, \mathrm{pol}}-q \Delta V_{\mathrm{OC}}$,

where $\Delta E_{\text {LUMO }}$ is the offset between donor and acceptor LUMO, which is required to split up excitons generated in the polymer. This offset is often required to be at least $0.3 \mathrm{eV}$ for efficient exciton dissociation (exceptions are, for example, reported in Ref. [96]) leading to an open-circuit voltage loss $\Delta V_{\mathrm{OC}}$ of at least $0.6 \mathrm{eV}$ compared to the polymer band gap. The polymer band gap also defines the portion of the sunlight that can be absorbed by the photoactive layer and thereby determines the short-circuit current

$$
J_{\mathrm{SC}}=q \int_{E_{g, \mathrm{pol}}}^{\infty} \mathrm{EQE} \times \Phi_{\mathrm{AM} 1.5}(E) d E
$$

under the assumption of a constant EQE (set to $65 \%$ in the Scharber paper). The standard solar photon flux spectrum irradiating on the solar cell is denoted as $\Phi_{\mathrm{AM} 1.5}$. By additionally setting the fill factor to $65 \%$, Scharber could calculate device efficiencies $\eta_{\text {Scharber }}$ from the polymer band gap and the LUMO offset (or, equivalently, $\Delta V_{\mathrm{OC}}$ ) which is depicted in Fig. 6(a). By entering values of devices with record efficiency at their time, the transition from P3HT towards polymers with lower band gaps and smaller LUMO offsets becomes evident. The driving factor in the development of organic solar cells with high-powerconversion efficiency (PCE) becomes clearer from Fig. 6(b), where the actual PCE (color scale) is related to the electronic quality $Q$ discussed in this work and a second figure of merit given by the efficiency potential $\eta_{\text {scharber }}$ of a material in the Scharber plot which indicates the degree of optimization of the polymer's energy levels. Although Scharber's analysis clearly focuses on the energy levels in the donor-acceptor blend and pays little attention 

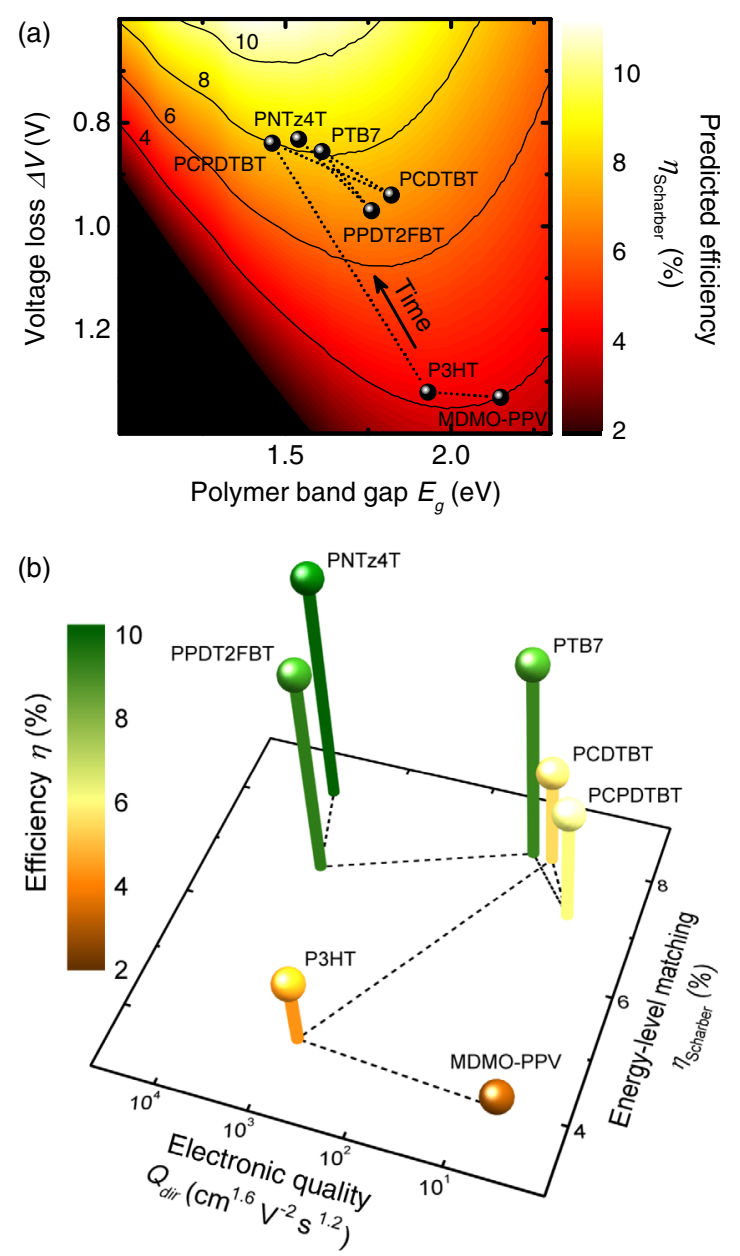

FIG. 6. (a) Predicted device efficiencies $\left(\eta_{\text {Scharber }}\right.$, color scale) calculated from polymer band gap and open-circuit voltage loss for an assumed EQE and FF of $65 \%$ following the work of Scharber et al. [19]. Devices that stated an efficiency record at their time are entered in the chart, which illustrates the successive development of polymers with optimized energy levels. A clear correlation between actual device efficiency (color scale) and $\eta_{\text {Scharber }}$ - which is ultimately a measure for favorable energylevel alignment - can be seen in (b), while no clear correlation to the electronic quality factor $Q$ is visible. Only the polymers PPDT2FBT [33] and PNTz4T [35] point towards the systematic improvement of electronic quality, which allows us to enhance device efficiency beyond the limits set in Scharber's work.

to the potential lying in higher values of $\mathrm{FF}$ and $\mathrm{EQE}$, it predicted the development of organic solar-cell efficiencies quite well up to $\mathrm{PTB} 7: \mathrm{PC}_{71} \mathrm{BM}$ [2], as can be seen from the clear correlation between $\eta_{\text {Scharber }}$ and PCE in Fig. 6(b). Naturally, this correlation cannot be exact because each of these records is a singular device and FFs and EQEs vary, mediated, for example, by different thicknesses as in the cases of poly[2,6-(4,4-bis-(2-ethylhexyl)-4 $H$-cyclopenta[2,1-b;3,4-b']-dithiophene)-alt-4,7-(2,1,3-benzothiadiazole)]: [6,6]-phenyl $\mathrm{C}_{71}$-butyric acid methyl ester (PCPDTBT:PC ${ }_{71} \mathrm{BM}$ ) [97] and PCDTBT: $\mathrm{PC}_{71} \mathrm{BM}$ [56]. On the other hand, there is no evident correlation between electronic quality $Q$ and $\mathrm{PCE}$. The clearest exception to these observations is P3HT: $\mathrm{PC}_{61} \mathrm{BM}$ [28], which increased the PCE from $2.5 \%$ to $4.4 \%$ compared to MDMO-PPV $: \mathrm{PC}_{61} \mathrm{BM}$ [1] while leaving $\eta_{\text {scharber }}$ almost unchanged. P3HT is well known for its high crystallinity leading to the large $Q$, which enabled a thick device with relatively high efficiency. Remarkably, the high- $Q$ polymer blend poly[(2,5-bis(2-hexyldecyloxy)phenylene)-alt-(5,6difluoro-4,7-di(thiophen-2-yl)benzo[c]-[1,2,5]thiadiazole)]: $[6,6]$-phenyl $\mathrm{C}_{71}$-butyric acid methyl ester (PPDT2FBT: $\mathrm{PC}_{71} \mathrm{BM}$ ) [33] achieves an efficiency slightly higher than that of PTB7 although its energy levels are clearly less favorable. For the future design of polymers, it is obvious that further advances in PCE need to take place in the top left corner of Fig. 6(b) where materials with optimized energy levels and high electronic quality are located. The recently published work on PNTz4T: $\mathrm{PC}_{71} \mathrm{BM}$ [35] has successfully taken a next step in this direction.

\section{APPLICATION TO OTHER MATERIAL CLASSES}

This study outlines a methodology based on driftdiffusion simulations which allows the definition of a charge-carrier collection coefficient with a unique relation to the (normalized) fill factor of a thin-film solar cell from a low-mobility material. We conduct our analysis for the highly relevant case of polymer-based organic solar cells. However, the presented methodology can be transferred to other materials in thin-film devices that have a $p$ - $i-n$-like geometry where a mostly homogeneous electrical field prevails in the photoactive layer. Materials that are rather new to the field of photovoltaics and that have not been characterized in depth are of special interest. Then the easyto-access electronic quality factor can be used to monitor material and device optimization and builds a basis to estimate the potential of a certain material. Thickness series provide further insight into whether a device is limited by an unfavorable architecture — such as energetically misaligned contacts - rather than the photoactive-layer material itself.

Among others, promising material systems used in thinfilm solar cells include perovskites and especially their lead-free alternatives, semiconducting nanoparticles such as $\mathrm{PbS}$ and $\mathrm{Bi}_{2} \mathrm{~S}_{3}$, and bilayers of $\mathrm{Sb}_{2} \mathrm{~S}_{3}$. The methodology presented in this work can be applied to all of these materials, and the relevant physical phenomena such as doping or unbalanced charge transport are the same. However, quantitative differences in the expression of the collection coefficient from the case of polymer solar cells can be expected for these materials because of their different dielectric constants.

\section{CONCLUSION}

In summary, our simulation-based studies provide a refined model for the complex behavior of the fill factor 
of organic solar cells for the limiting situations of trapassisted and band-to-band recombination. We find an explicit expression for the electronic quality of the active-layer material which governs the fill factor. By normalizing the fill factor $\mathrm{FF}_{n}$ with respect to the opencircuit voltage and using an optimized collection coefficient $\gamma$, we achieve a clearer mapping between $\mathrm{FF}_{n}$ and $\gamma$ compared to the previous work of Bartesaghi et al. This allows us to accurately determine the electronic quality factor $Q$ from nothing more than FF, open-circuit voltage $V_{\mathrm{OC}}$, short-circuit current density $J_{\mathrm{SC}}$, and active-layer thickness. All these measurements are routinely done in organic solar-cell research, which makes $Q$ extraction feasible to be part of standard device characterization protocols and allows the extensive study of the literature data in retrospect. The simplicity of this analysis is particularly striking, considering that the direct access of the quantities that constitute $Q$ (mobility and lifetime or recombination coefficient) is cumbersome and depends heavily on the measurement conditions. For reliable $Q$ extraction, our model assumes a homogeneous electrical field. For thick devices, doping or unbalanced charge transport lead to the formation of an electric-field-free neutral zone where carrier collection is poor but independent of applied voltage which heavily deteriorates $J_{\mathrm{SC}}$ and device efficiency while slightly higher FFs are reached compared to the fully depleted case. Devices with good collection coefficient $\gamma$ and high FFs remain unaffected. Contrary to this, the contact layers mainly affect the highest obtainable FFs. External series resistances and Schottky barriers to the contacts lower the FF, while low-surfacerecombination velocities of minority carriers lead to an unprecedented improvement of the FF.

From an experimental point of view, preparing a series of devices with varying active-layer thickness-which is commonly done during device optimization-and analyzing the thickness dependence of the FF provides important information about device properties such as space-charge regions and contacts in addition to the quantification of the material's electronic quality. Because this analysis requires little effort, it is valuable as a first indicator for the mentioned properties which can then be followed up by detailed quantitative characterization, for example, voltagedependent capacitance measurements that determine the exact doping level. A unique advantage of the presented method is that its retrospective character enables the reevaluation of previously recorded data from thickness scans.

Finally, we illustrate that the paradigm for increasing organic solar-cell efficiencies by optimizing the energy levels of the polymer - namely, band gap and LUMO offset - has been very successful in the past and has brought up polymers that are energetically close to the optimum. However, further advances in conjugated polymer-based energy harvesting demand enhanced carrier collection for devices with thicknesses around the second absorption maximum of 200-250 nm implying higher values of external quantum efficiency and fill factor. This insight sets the need for polymers that combine optimized energy levels with high electronic quality. First steps toward such ordered crystalline polymers have been accomplished recently. The easy-to-access electronic quality factor specified in this work is a valuable figure of merit that can be used to monitor the further progress towards highly efficient organic solar cells.

\section{ACKNOWLEDGMENTS}

We acknowledge support from the Deutsche Forschungsgemeinschaft (DFG) (Grants No. KI-1571/2-1 and No. RA 473/7-1).

[1] S. E. Shaheen, C. J. Brabec, N. S. Sariciftci, F. Padinger, T. Fromherz, and J.C. Hummelen, $2.5 \%$ efficient organic plastic solar cells, Appl. Phys. Lett. 78, 841 (2001).

[2] Z. He, C. Zhong, S. Su, M. Xu, H. Wu, and Y. Cao, Enhanced power-conversion efficiency in polymer solar cells using an inverted device structure, Nat. Photonics 6, 593 (2012).

[3] E. Bundgaard and F. Krebs, Low band gap polymers for organic photovoltaics, Sol. Energy Mater. Sol. Cells 91, 954 (2007).

[4] R. Kroon, M. Lenes, J. C. Hummelen, P. W. M. Blom, and B. de Boer, Small bandgap polymers for organic solar cells (polymer material development in the last 5 years), Polym. Rev. 48, 531 (2008).

[5] G. Li, R. Zhu, and Y. Yang, Polymer solar cells, Nat. Photonics 6, 153 (2012).

[6] C. Liu, K. Wang, X. Gong, and A. J. Heeger, Low bandgap semiconducting polymers for polymeric photovoltaics, Chem. Soc. Rev. (2016).

[7] J. Roncali, Molecular engineering of the band gap of pi-conjugated systems: Facing technological applications, Macromol. Rapid Commun. 28, 1761 (2007).

[8] M. A. Faist, T. Kirchartz, W. Gong, R. S. Ashraf, I. McCulloch, J. C. de Mello, N. J. Ekins-Daukes, D. D. C. Bradley, and J. Nelson, Competition between the charge transfer state and the singlet states of donor or acceptor limiting the efficiency in polymer:fullerene solar cells, J. Am. Chem. Soc. 134, 685 (2012).

[9] M. A. Faist, S. Shoaee, S. Tuladhar, G. F. A. Dibb, S. Foster, W. Gong, T. Kirchartz, D. D. C. Bradley, J. R. Durrant, and J. Nelson, Understanding the reduced efficiencies of organic solar cells employing fullerene multiadducts as acceptors, Adv. Energy Mater. 3, 744 (2013).

[10] G. Zhao, Y. He, and Y. Li, 6.5\% efficiency of polymer solar cells based on poly(3-hexylthiophene) and indene- $\mathrm{C}_{60}$ bisadduct by device optimization, Adv. Mater. 22, 4355 (2010).

[11] S. Holliday, R. S. Ashraf, C. B. Nielsen, M. Kirkus, J. A. Röhr, C.-H. Tan, E. Collado-Fregoso, A.-C. Knall, J. R. Durrant, J. Nelson, and I. McCulloch, A rhodanine flanked 
nonfullerene acceptor for solution-processed organic photovoltaics, J. Am. Chem. Soc. 137, 898 (2015).

[12] D. Meng, D. Sun, C. Zhong, T. Liu, B. Fan, L. Huo, Y. Li, W. Jiang, H. Choi, T. Kim, J. Y. Kim, Y. Sun, Z. Wang, and A.J. Heeger, High-performance solutionprocessed non-fullerene organic solar cells based on selenophene-containing perylene bisimide acceptor, J. Am. Chem. Soc. 138, 375 (2016).

[13] C. B. Nielsen, S. Holliday, H.-Y. Chen, S. J. Cryer, and I. McCulloch, Non-fullerene electron acceptors for use in organic solar cells, Acc. Chem. Res. 48, 2803 (2015).

[14] C. B. Nielsen, E. Voroshazi, S. Holliday, K. Cnops, D. Cheyns, and I. McCulloch, Electron-deficient truxenone derivatives and their use in organic photovoltaics, J. Mater. Chem. A 2, 12348 (2014).

[15] H.-Y. Chen, J. Hou, S. Zhang, Y. Liang, G. Yang, Y. Yang, L. Yu, Y. Wu, and G. Li, Polymer solar cells with enhanced open-circuit voltage and efficiency, Nat. Photonics 3, 649 (2009).

[16] J. Hou, M.-H. Park, S. Zhang, Y. Yao, L.-M. Chen, J.-H. Li, and Y. Yang, Bandgap and molecular energy level control of conjugated polymer photovoltaic materials based on benzo[1,2-b:4,5-b']dithiophene, Macromolecules 41, 6012 (2008).

[17] T. Ishwara, D. D. C. Bradley, J. Nelson, P. Ravirajan, I. Vanseveren, T. Cleij, D. Vanderzande, L. Lutsen, S. Tierney, M. Heeney, and I. McCulloch, Influence of polymer ionization potential on the open-circuit voltage of hybrid polymer/TiO 2 solar cells, Appl. Phys. Lett. 92, 053308 (2008).

[18] W. Li, K. H. Hendriks, A. Furlan, M. M. Wienk, and R. A. J. Janssen, High quantum efficiencies in polymer solar cells at energy losses below 0.6 eV, J. Am. Chem. Soc. 137, 2231 (2015).

[19] M. C. Scharber, D. Mühlbacher, M. Koppe, P. Denk, C. Waldauf, A. J. Heeger, and C. J. Brabec, Design rules for donors in bulk-heterojunction solar cells-towards $10 \%$ energy-conversion efficiency, Adv. Mater. 18, 789 (2006).

[20] M. A. Green, Accuracy of analytical expressions for solar cell fill factors, Sol. Cells 7, 337 (1982).

[21] H. Hoppe, S. Shokhovets, and G. Gobsch, Inverse relation between photocurrent and absorption layer thickness in polymer solar cells, Phys. Status Solidi RRL 1, R40 (2007).

[22] A. J. Moulé, J. B. Bonekamp, and K. Meerholz, The effect of active layer thickness and composition on the performance of bulk-heterojunction solar cells, J. Appl. Phys. 100, 094503 (2006).

[23] H. Hoppe, N. Arnold, N. S. Sariciftci, and D. Meissner, Modeling the optical absorption within conjugated polymer/ fullerene-based bulk-heterojunction organic solar cells, Sol. Energy Mater. Sol. Cells 80, 105 (2003).

[24] J. A. Bartelt, Z. M. Beiley, E. T. Hoke, W. R. Mateker, J. D. Douglas, B. A. Collins, J. R. Tumbleston, K. R. Graham, A. Amassian, H. Ade, J. M. J. Fréchet, M. F. Toney, and M. D. McGehee, The importance of fullerene percolation in the mixed regions of polymer-fullerene bulk heterojunction solar cells, Adv. Energy Mater. 3, 364 (2013).

[25] S. Foster, F. Deledalle, A. Mitani, T. Kimura, K.-B. Kim, T. Okachi, T. Kirchartz, J. Oguma, K. Miyake, J. R. Durrant,
S. Doi, and J. Nelson, Electron collection as a limit to polymer:PCBM solar cell efficiency: Effect of blend microstructure on carrier mobility and device performance in PTB7:PCBM, Adv. Energy Mater. 4, 1400311 (2014).

[26] M. Lenes, L. J. A. Koster, V. D. Mihailetchi, and P. W. M. Blom, Thickness dependence of the efficiency of polymer: fullerene bulk heterojunction solar cells, Appl. Phys. Lett. 88, 243502 (2006).

[27] J. Peet, L. Wen, P. Byrne, S. Rodman, K. Forberich, Y. Shao, N. Drolet, R. Gaudiana, G. Dennler, and D. Waller, Bulk heterojunction solar cells with thick active layers and high fill factors enabled by a bithiophene-co-thiazolothiazole push-pull copolymer, Appl. Phys. Lett. 98, 043301 (2011).

[28] G. Li, V. Shrotriya, J. Huang, Y. Yao, T. Moriarty, K. Emery, and Y. Yang, High-efficiency solution processable polymer photovoltaic cells by self-organization of polymer blends, Nat. Mater. 4, 864 (2005).

[29] Z. Chen, P. Cai, J. Chen, X. Liu, L. Zhang, L. Lan, J. Peng, Y. Ma, and Y. Cao, Low band-gap conjugated polymers with strong interchain aggregation and very high hole mobility towards highly efficient thick-film polymer solar cells, Adv. Mater. 26, 2586 (2014).

[30] X. Guo, N. Zhou, S. J. Lou, J. Smith, D. B. Tice, J. W. Hennek, R. P. Ortiz, J. T. L. Navarrete, S. Li, J. Strzalka, L. X. Chen, R. P. H. Chang, A. Facchetti, and T. J. Marks, Polymer solar cells with enhanced fill factors, Nat. Photonics 7, 825 (2013).

[31] W. Li, K. H. Hendriks, A. Furlan, W. S. C. Roelofs, M. M. Wienk, and R. A. J. Janssen, Universal correlation between fibril width and quantum efficiency in diketopyrrolopyrrolebased polymer solar cells, J. Am. Chem. Soc. 135, 18942 (2013).

[32] Y. Liu, J. Zhao, Z. Li, C. Mu, W. Ma, H. Hu, K. Jiang, H. Lin, H. Ade, and H. Yan, Aggregation and morphology control enables multiple cases of high-efficiency polymer solar cells, Nat. Commun. 5, 5293 (2014).

[33] T. L. Nguyen, H. Choi, S.-J. Ko, M. A. Uddin, B. Walker, S. Yum, J.-E. Jeong, M. H. Yun, T. J. Shin, S. Hwang, J. Y. Kim, and H. Y. Woo, Semi-crystalline photovoltaic polymers with efficiency exceeding $9 \%$ in a $300 \mathrm{~nm}$ thick conventional single-cell device, Energy Environ. Sci. 7, 3040 (2014).

[34] S. C. Price, A. C. Stuart, L. Yang, H. Zhou, and W. You, Fluorine substituted conjugated polymer of medium band gap yields $7 \%$ efficiency in polymer-fullerene solar cells, J. Am. Chem. Soc. 133, 4625 (2011).

[35] V. Vohra, K. Kawashima, T. Kakara, T. Koganezawa, I. Osaka, K. Takimiya, and H. Murata, Efficient inverted polymer solar cells employing favourable molecular orientation, Nat. Photonics 9, 403 (2015).

[36] M. Zhang, X. Guo, W. Ma, H. Ade, and J. Hou, A polythiophene derivative with superior properties for practical application in polymer solar cells, Adv. Mater. 26, 5880 (2014).

[37] T. Kirchartz, T. Agostinelli, M. Campoy-Quiles, W. Gong, and J. Nelson, Understanding the thickness-dependent performance of organic bulk heterojunction solar cells: The influence of mobility, lifetime, and space charge, J. Phys. Chem. Lett. 3, 3470 (2012).

[38] D. Bartesaghi, I. del Carmen Pérez, J. Kniepert, S. Roland, M. Turbiez, D. Neher, and L. J. A. Koster, Competition 
between recombination and extraction of free charges determines the fill factor of organic solar cells, Nat. Commun. 6, 7083 (2015).

[39] R. C. I. MacKenzie, C. G. Shuttle, M. L. Chabinyc, and J. Nelson, Extracting microscopic device parameters from transient photocurrent measurements of P3HT:PCBM solar cells, Adv. Energy Mater. 2, 662 (2012).

[40] S. Schäfer, A. Petersen, T. A. Wagner, R. Kniprath, D. Lingenfelser, A. Zen, T. Kirchartz, B. Zimmermann, U. Würfel, X. Feng, and T. Mayer, Influence of the indium tin oxide/organic interface on open-circuit voltage, recombination, and cell degradation in organic small-molecule solar cells, Phys. Rev. B 83, 165311 (2011).

[41] W. Tress, K. Leo, and M. Riede, Influence of hole-transport layers and donor materials on open-circuit voltage and shape of $I-V$ curves of organic solar cells, Adv. Funct. Mater. 21, 2140 (2011).

[42] U. Würfel, D. Neher, A. Spies, and S. Albrecht, Impact of charge transport on current-voltage characteristics and power-conversion efficiency of organic solar cells, Nat. Commun. 6, 6951 (2015).

[43] L. J. A. Koster, V. D. Mihailetchi, and P. W. M. Blom, Bimolecular recombination in polymer/fullerene bulk heterojunction solar cells, Appl. Phys. Lett. 88, 052104 (2006).

[44] M. M. Mandoc, F. B. Kooistra, J. C. Hummelen, B. de Boer, and P. W. M. Blom, Effect of traps on the performance of bulk heterojunction organic solar cells, Appl. Phys. Lett. 91, 263505 (2007).

[45] R. A. Street, A. Krakaris, and S. R. Cowan, Recombination through different types of localized states in organic solar cells, Adv. Funct. Mater. 22, 4608 (2012).

[46] T. Kirchartz and J. Nelson, Device modelling of organic bulk heterojunction solar cells, Top. Curr. Chem. 352, 279 (2014).

[47] T. Kirchartz, B. E. Pieters, J. Kirkpatrick, U. Rau, and J. Nelson, Recombination via tail states in polythiophene: fullerene solar cells, Phys. Rev. B 83, 115209 (2011).

[48] T. Kirchartz, B. E. Pieters, K. Taretto, and U. Rau, Electrooptical modeling of bulk heterojunction solar cells, J. Appl. Phys. 104, 094513 (2008).

[49] L. J. A. Koster, E. C. P. Smits, V. D. Mihailetchi, and P. W. M. Blom, Device model for the operation of polymer/ fullerene bulk heterojunction solar cells, Phys. Rev. B 72, 085205 (2005).

[50] G. F. Burkhard, E. T. Hoke, and M. D. McGehee, Accounting for interference, scattering, and electrode absorption to make accurate internal quantum efficiency measurements in organic and other thin solar cells, Adv. Mater. 22, 3293 (2010).

[51] L. A. A. Pettersson, L. S. Roman, and O. Inganäs, Modeling photocurrent action spectra of photovoltaic devices based on organic thin films, J. Appl. Phys. 86, 487 (1999).

[52] See Supplemental Material at http://link.aps.org/ supplemental/10.1103/PhysRevApplied.6.024001 for more experimental data from the literature, further simulation details, and results for the statistical ensembles of devices.

[53] C. Deibel, A. Baumann, and V. Dyakonov, Polaron recombination in pristine and annealed bulk heterojunction solar cells, Appl. Phys. Lett. 93, 163303 (2008).
[54] G. Lakhwani, A. Rao, and R. H. Friend, Bimolecular recombination in organic photovoltaics, Annu. Rev. Phys. Chem. 65, 557 (2014).

[55] Y. Liang, Z. Xu, J. Xia, S.-T. Tsai, Y. Wu, G. Li, C. Ray, and $\mathrm{L}$. Yu, For the bright future-bulk heterojunction polymer solar cells with power conversion efficiency of $7.4 \%$, Adv. Mater. 22, E135 (2010).

[56] S. H. Park, A. Roy, S. Beaupré, S. Cho, N. Coates, J. S. Moon, D. Moses, M. Leclerc, K. Lee, and A. J. Heeger, Bulk heterojunction solar cells with internal quantum efficiency approaching 100\%, Nat. Photonics 3, 297 (2009).

[57] C. M. Proctor, C. Kim, D. Neher, and T.-Q. Nguyen, Nongeminate recombination and charge transport limitations in diketopyrrolopyrrole-based solutionprocessed small molecule solar cells, Adv. Funct. Mater. 23, 3584 (2013).

[58] C. G. Shuttle, B. O’Regan, A. M. Ballantyne, J. Nelson, D. D. C. Bradley, and J. R. Durrant, Bimolecular recombination losses in polythiophene:fullerene solar cells, Phys. Rev. B 78, 113201 (2008).

[59] D. Credgington, F. C. Jamieson, B. Walker, T.-Q. Nguyen, and J. R. Durrant, Quantification of geminate and nongeminate recombination losses within a solution-processed small-molecule bulk heterojunction solar cell, Adv. Mater. 24, 2135 (2012).

[60] J. Kniepert, I. Lange, J. Heidbrink, J. Kurpiers, T. J. K. Brenner, L. J. A. Koster, and D. Neher, Effect of solvent additive on generation, recombination, and extraction in PTB7:PCBM solar cells: A conclusive experimental and numerical simulation study, J. Phys. Chem. C 119, 8310 (2015).

[61] A. Maurano, R. Hamilton, C. G. Shuttle, A. M. Ballantyne, J. Nelson, B. O'Regan, W. Zhang, I. McCulloch, H. Azimi, M. Morana, C. J. Brabec, and J. R. Durrant, Recombination dynamics as a key determinant of open circuit voltage in organic bulk heterojunction solar cells: A comparison of four different donor polymers, Adv. Mater. 22, 4987 (2010).

[62] G. Juska, K. Arlauskas, J. Stuchlik, and R. Österbacka, NonLangevin bimolecular recombination in low-mobility materials, J. Non-Cryst. Solids 352, 1167 (2006).

[63] D. Neher, J. Kniepert, A. Elimelech, and L. J. A. Koster, A new figure of merit for organic solar cells with transportlimited photocurrents, Sci. Rep. 6, 24861 (2016).

[64] G. Garcia-Belmonte, A. Munar, E. M. Barea, J. Bisquert, I. Ugarte, and R. Pacios, Charge carrier mobility and lifetime of organic bulk heterojunctions analyzed by impedance spectroscopy, Org. Electron. 9, 847 (2008).

[65] V. D. Mihailetchi, J. Wildeman, and P. W. M. Blom, SpaceCharge Limited Photocurrent, Phys. Rev. Lett. 94, 126602 (2005).

[66] M. Stolterfoht, A. Armin, B. Philippa, R. D. White, P. L. Burn, P. Meredith, G. Juska, and A. Pivrikas, Photocarrier drift distance in organic solar cells and photodetectors, Sci. Rep. 5, 9949 (2015).

[67] R. S. Crandall, Transport in hydrogenated amorphous silicon $p-i-n$ solar cells, J. Appl. Phys. 53, 3350 (1982).

[68] R. S. Crandall and I. Balberg, Mobility-lifetime products in hydrogenated amorphous silicon, Appl. Phys. Lett. 58, 508 (1991). 
[69] C. Deibel, A. Wagenpfahl, and V. Dyakonov, Influence of charge carrier mobility on the performance of organic solar cells, Phys. Status Solidi RRL 2, 175 (2008).

[70] W. Tress, K. Leo, and M. Riede, Optimum mobility, contact properties, and open-circuit voltage of organic solar cells: A drift-diffusion simulation study, Phys. Rev. B 85, 155201 (2012).

[71] K. Vandewal, K. Tvingstedt, A. Gadisa, O. Inganäs, and J. V. Manca, On the origin of the open-circuit voltage of polymer-fullerene solar cells, Nat. Mater. 8, 904 (2009).

[72] M. A. Green, Accurate expressions for solar cell fill factors including series and shunt resistances, Appl. Phys. Lett. 108, 081111 (2016).

[73] M. A. Green, Accuracy of analytical expressions for solar cell fill factors, Sol. Cells 7, 337 (1982).

[74] K. Hecht, Zum mechanismus des lichtelektrischen primärstromes in isolierenden kristallen, Z. Phys. 77, 235 (1932).

[75] A. Baumann, J. Lorrmann, D. Rauh, C. Deibel, and V. Dyakonov, A new approach for probing the mobility and lifetime of photogenerated charge carriers in organic solar cells under real operating conditions, Adv. Mater. 24, 4381 (2012).

[76] A. Pivrikas, N. S. Sariciftci, G. Juska, and R. Österbacka, A review of charge transport and recombination in polymer/ fullerene organic solar cells, Prog. Photovoltaics 15, 677 (2007).

[77] M. A. Green, Solar cell fill factors: General graph and empirical expressions, Solid State Electron. 24, 788 (1981).

[78] O. J. Sandberg, M. Nyman, and R. Österbacka, Effect of Contacts in Organic Bulk Heterojunction Solar Cells, Phys. Rev. Applied 1, 024003 (2014).

[79] Z. Tang, W. Tress, Q. Bao, M. J. Jafari, J. Bergqvist, T. Ederth, M. R. Andersson, and O. Inganäs, Improving cathodes with a polymer interlayer in reversed organic solar cells, Adv. Energy Mater. 4, 1400643 (2014).

[80] A. G. Aberle, Surface passivation of crystalline silicon solar cells: A review, Prog. Photovoltaics 8, 473 (2000).

[81] F. Deledalle, T. Kirchartz, M. S. Vezie, M. Campoy-Quiles, P. Shakya Tuladhar, J. Nelson, and J. R. Durrant, Understanding the Effect of Unintentional Doping on Transport Optimization and Analysis in Efficient Organic BulkHeterojunction Solar Cells, Phys. Rev. X 5, 011032 (2015).

[82] G. F. A. Dibb, M.-A. Muth, T. Kirchartz, S. Engmann, H. Hoppe, G. Gobsch, M. Thelakkat, N. Blouin, S. Tierney, M. Carrasco-Orozco, J. R. Durrant, and J. Nelson, Influence of doping on charge carrier collection in normal and inverted geometry polymer:fullerene solar cells, Sci. Rep. 3, 3335 (2013).

[83] T. Kirchartz and J. Nelson, Meaning of reaction orders in polymer:fullerene solar cells, Phys. Rev. B 86, 165201 (2012).
[84] C. M. Proctor, J. A. Love, and T.-Q. Nguyen, Mobility guidelines for high fill factor solution-processed small molecule solar cells, Adv. Mater. 26, 5957 (2014).

[85] J. G. Tait, U. W. Paetzold, D. Cheyns, M. Turbiez, P. Heremans, and B. P. Rand, Interfacial depletion regions: Beyond the space charge limit in thick bulk heterojunctions, ACS Appl. Mater. Interfaces 8, 2211 (2016).

[86] V. A. Trukhanov, V. V. Bruevich, and D. Y. Paraschuk, Effect of doping on performance of organic solar cells, Phys. Rev. B 84, 205318 (2011).

[87] H. Azimi, A. Senes, M. C. Scharber, K. Hingerl, and C. J. Brabec, Charge transport and recombination in lowbandgap bulk heterojunction solar cell using bis-adduct fullerene, Adv. Energy Mater. 1, 1162 (2011).

[88] J. Bisquert and G. Garcia-Belmonte, On voltage, photovoltage, and photocurrent in bulk heterojunction organic solar cells, J. Phys. Chem. Lett. 2, 1950 (2011).

[89] G. Garcia-Belmonte, A. Guerrero, and J. Bisquert, Elucidating operating modes of bulk-heterojunction solar cells from impedance spectroscopy analysis, J. Phys. Chem. Lett. 4, 877 (2013).

[90] G. Dicker, M. P. de Haas, J. M. Warman, D. M. de Leeuw, and L. D. A. Siebbeles, The disperse charge-carrier kinetics in regioregular poly(3-hexylthiophene), J. Phys. Chem. B 108, 17818 (2004).

[91] Z. Liang, A. Nardes, D. Wang, J. J. Berry, and B. A. Gregg, Defect engineering in pi-conjugated polymers, Chem. Mater. 21, 4914 (2009).

[92] H.-H. Liao, C.-M. Yang, C.-C. Liu, S.-F. Horng, H.-F. Meng, and J.-T. Shy, Dynamics and reversibility of oxygen doping and de-doping for conjugated polymer, J. Appl. Phys. 103, 104506 (2008).

[93] V. D. Mihailetchi, H. X. Xie, B. de Boer, L. J. A. Koster, and P.W.M. Blom, Charge transport and photocurrent generation in poly(3-hexylthiophene): Methanofullerene bulk-heterojunction solar cells, Adv. Funct. Mater. 16, 699 (2006).

[94] S. Schiefer, B. Zimmermann, and U. Würfel, Determination of the intrinsic and the injection dependent charge carrier density in organic solar cells using the Suns-VOC method, J. Appl. Phys. 115, 044506 (2014).

[95] M. C. Scharber, On the efficiency limit of conjugated polymer:fullerene-based bulk heterojunction solar cells, Adv. Mater. 28, 1994 (2016).

[96] K. Vandewal, Z. Ma, J. Bergqvist, Z. Tang, E. Wang, P. Henriksson, K. Tvingstedt, M. R. Andersson, F. Zhang, and O. Inganäs, Quantification of quantum efficiency and energy losses in low bandgap polymer:fullerene solar cells with high open-circuit voltage, Adv. Funct. Mater. 22, 3480 (2012).

[97] J. Peet, J. Y. Kim, N. E. Coates, W. L. Ma, D. Moses, A. J. Heeger, and G. C. Bazan, Efficiency enhancement in lowbandgap polymer solar cells by processing with alkane dithiols, Nat. Mater. 6, 497 (2007). 